\title{
LIBERALIZACIÓN COMERCIAL EN COLOMBIA: ¿MODERNIZACIÓN O TRAMPA?"
}

\author{
Jorge Coronel-Lópeza \\ Jenny Paola Danna-Buitrago ${ }^{\mathrm{b}}$ \\ Álvaro Mercado-Suárezc
}

" DOI: https://doi.org/10.18601/01245996.v21n41.04. Recepción: 27-062017, modificación final: 01-04-2019, aceptación: 14-05-2019. Sugerencia de citación: Coronel-L. J., Danna-B., J. y Mercado-S., A. (2019). Liberalización comercial en Colombia: ¿Modernización o trampa? Revista de Economía Institucional, 21(41), 71-97.

a Magíster en Economía. Profesor Investigador, Universidad de $\mathrm{Me}$ dellín, Colombia, Investigador externo de la Fundación Universitaria Los Libertadores, y miembro del Observatorio Colombiano de Tratados Comerciales (OCTC), Bogotá, Colombia, [jecorone1@udem.edu.co], [https://orcid.org/0000-0001-8811-0295].

b PhD. en Ciencias Económicas. Directora de Investigación en Globalización y Desarrollo Sostenible, Directora Observatorio Colombiano de Tratados Comerciales (OCTC), Fundación Universitaria Los Libertadores, Bogotá, Colombia, [jenny.danna@libertadores.edu.co], [https://orcid.org/0000-0003-0241-9481].

c Magíster en Desarrollo Regional. Decano Facultad Ciencias Económicas, Administrativas y Contables, Fundación Universitaria Los Libertadores, Bogotá, Colombia, [alvaro.mercado@libertadores.edu.co], [https://orcid.org/0000-0002-9928-0190]. 


\section{Liberalización comercial en Colombia: ¿modernización o trampa?}

Resumen Este artículo analiza el comercio exterior colombiano para determinar por qué los resultados de la apertura no han sido satisfactorios frente a los propósitos que la impulsaron. Se plantea como hipótesis que el modelo de desarrollo está sujeto a dicha política y que la liberalización comercial no ha motivado el desarrollo de sectores que agreguen valor. Finalmente, mediante la teoría de los ciclos políticos, se sugiere que la economía colombiana puede estar atrapada en una trampa de dependencia económica internacional a la que ha contribuido la dirigencia política del país.

Palabras clave: liberalización comercial, Colombia, ciclos políticos de los negocios, comercio internacional; JEL: F13, F14

\section{Trade liberalization in Colombia: modernization or pitfall?}

Abstract This paper discusses the gap between the expected and real effects of Colombian trade liberalization policy. First, we stress the influence of international integration on Colombia's growth and development, and explains why moving toward more free trade has not contributed to the consolidation of high value-added industries in that country. Ultimately, we apply the theory of political cycles and suggest that political power in Colombia has contributed to get the country trapped into an international dependency on primary commodities.

Keywords: trade liberalization, Colombia, political business cycles, international trade; JEL: F13, F14

\section{Liberalização comercial na Colômbia: modernização ou armadilha?}

Resumo Este artigo analisa o comércio exterior colombiano para determinar por que os resultados da abertura não foram satisfatórios em vista dos objetivos que o conduziram. Hipotetiza-se que o modelo de desenvolvimento está sujeito a essa política e que a liberalização do comércio não motivou o desenvolvimento de setores que agregam valor. Finalmente, através da teoria dos ciclos políticos, sugere-se que a economia colombiana possa estar presa em uma armadilha de dependência econômica internacional para a qual a liderança política do país contribuiu.

Palavras-chaves: liberalização comercial, Colômbia, ciclos políticos de negócios, comércio internacional; JEL: F13, F14 
D esde 1990 se adoptó en Colombia una política de liberalización comercial para modernizar la economía, ampliar la oferta exportable, insertarse en los mercados internacionales y lograr estabilidad macroeconómica, conocida como apertura económica (Ocampo y Villar, 1992). Ya han pasado casi 30 años y el país no ha diversificado sus exportaciones, se ha estancado el crecimiento y no ha fortalecido los sectores que agregan valor (García, 2002; López, López y Montes, 2015). ¿Qué aspectos pueden explicar esta situación? Quizá la respuesta desborda las fronteras de la ciencia económica; sin embargo, se presentan algunas respuestas posibles.

Desde entonces las políticas de desarrollo han procurado liberalizar la economía y han expuesto el desarrollo del país a los vaivenes de la apertura. Si bien la apertura no es buena ni mala per se, las políticas pueden orientar la economía hacia nuevas actividades que aprovechen las ventajas de la liberalización o a que mantenga viejas actividades y exporte bienes tradicionales. En Colombia, la liberalización ha llevado a que su economía gravite alrededor de la demanda externa de bienes primarios sin valor agregado y no a diversificar las exportaciones. ¿Quién ha influido en esta decisión? ¿Cuál ha sido la influencia de los sectores privado y público? Una posible hipótesis es que el país está atrapado en un modelo de desarrollo que impide mantener una senda de crecimiento estable, modernizar la estructura productiva y diversificar las exportaciones, debido a la inclinación a explotar recursos naturales que reportan altos ingresos en épocas de buenos precios internacionales.

Se puede decir, entonces, que la apertura ha fracasado a la luz de sus fines: Quizá solo ha conseguido que la economía siga sometida a las actividades primarias, extractivas y de bienes básicos. Así, es posible que se encuentre en una trampa de dependencia internacional. Para respaldar esta hipótesis, la primera sección resume la política de liberalización comercial colombiana. La segunda sintetiza algunas consideraciones teóricas sobre los ciclos políticos de los negocios (CPN). La tercera establece la existencia de una propensión (des) favorable a la liberalización comercial y al crecimiento económico dependiendo el partido político a la cabeza del diseño de políticas. La cuarta analiza los principales cambios macroeconómicos después de la liberalización comercial. Por último, se presentan las conclusiones. 


\section{LA POLÍTICA DE LIBERALIZACIÓN COMERCIAL EN COLOMBIA}

Después de la crisis de la deuda en los años ochenta, los países latinoamericanos tuvieron que implementar las reformas de estabilización económica y ajuste estructural consignadas en el Consenso de Washington (Williamson, 1990). Esto marcó el regreso de las políticas neoliberales que pretendían lograr el desarrollo de estos endeudados países conforme a los programas de la instituciones financieras internacionales (Berr y Combarnous, 2004). Sin embargo, más allá de lograr su desarrollo, el propósito de esos programas era apresurar la liberalización comercial para que elevaran las exportaciones, según sus ventajas comparativas, y así obtuvieran divisas para reembolsar la deuda (Berr, 2003).

Otros propósitos de la liberalización eran cambiar el modelo económico proteccionista que había creado problemas estructurales (Edwards, 1994) y limitar el papel económico del Estado, que habría intentado una industrialización forzosa siguiendo el modelo de industrialización por sustitución de importaciones (ISI) (Bejarano, 1998). Ahora, el Estado debía dejar vía libre a las iniciativas de los agentes para maximizar la riqueza, lo que traería como efecto residual su distribución a toda la población (Stiglitz, 2010). Solo debía intervenir para corregir las externalidades negativas o cuando estuviesen en peligro los bienes públicos.

Los cambios centrados en la autorregulación de los mercados mejorarían la eficiencia de las empresas (Gelbard, 1990). La liberalización aumentaría la competencia, incitando a los productores a aprovechar sus ventajas comparativas. Se esperaba entonces un uso más eficiente de los recursos y que parte de ellos se liberara para producir más y a menor costo, lo que reduciría el precio de venta y consolidaría dichas ventajas (Balassa, 1985). Con esa consolidación, los productores nacionales tendrían mejores oportunidades en los mercados internacionales, lo que favorecería aún más las exportaciones y, con ello, el aumento de la producción (McKinnon, 1964). Las mayores divisas permitirían importar bienes de capital que incorporaran el progreso técnico y redujeran los precios, mejorando la competitividad internacional. "las exportaciones se estimulan, así como la producción [...] y así sucesivamente según el círculo virtuoso de export-led growth” (Danna-B., 2012, p. 13).

En Colombia, la liberalización comercial se basó en la necesidad de promover la modernización del aparato productivo y el crecimiento. 
En el plan de desarrollo del presidente Gaviria (1990-1994) se argumentó que la apertura fue el camino recorrido por los países que eran potencias mundiales, pues el crecimiento de las exportaciones permitió su modernización y les garantizó un puesto en el mercado internacional (DNP, 1991). Se hicieron entonces diversas reformas de estabilización económica y ajuste estructural: reformas legales para desregular la economía, reformas tributarias y de gasto público, flexibilización del régimen laboral, reforma de la seguridad social, privatización de empresas públicas, reforma de la Constitución, etc.

Además, se redujeron las barreras arancelarias y paraarancelarias. Según la Cámara de Comercio de Bogotá (1993), entre 1990-1993 se eliminaron los controles administrativos y la sobretasa a las importaciones; $y$ se redujeron los aranceles de insumos y bienes de capital. $\mathrm{E}$ l arancel promedio pasó del $26,8 \%$ al 11,8\% y la protección efectiva promedio del 44\% al 24,8\%. Lora (2001) presenta algunos datos más: en 1989 los derechos de aduana eran en promedio del 47,6\% y en 1991 habían descendido al 6,7\%.

La estructura arancelaria también se racionalizó con el argumento de integrarse a la economía mundial, acceder a nuevos mercados, crear condiciones favorables para aumentar la competitividad y dinamizar las exportaciones (OMC, 1996; Echavarría, 2001). Para lograr así un crecimiento mayor y sostenido que redujera el desempleo, la pobreza y la desigualdad. La liberalización contribuiría al desarrollo, afirmación que se sustentó en las primeras teorías del desarrollo que lo ven como un resultado automático del crecimiento (Rostow, 1963), y generaría externalidades positivas como la diversificación de las exportaciones, lo que llevaría a una mayor estabilidad de los ingresos por exportaciones, que ya no dependerían de bienes básicos con precios volátiles, a un mayor crecimiento económico de largo plazo debido a tal estabilidad y un mayor dinamismo comercial debido a la ampliación de la base exportable (CNUCED, 2003; Dingemans y Ross, 2012).

No obstante, esa argumentación no consideró tres aspectos fundamentales que ayudan a entender por qué no se han obtenido esos resultados. Primero, desconoció la bilateralidad del comercio y subestimó la competencia internacional, pues suponía que la eliminación de las barreras beneficiaría automáticamente las exportaciones frente a las importaciones. Segundo, ignoró que el crecimiento no puede resolver problemas como el desempleo, la pobreza y la desigualdad sin políticas redistributivas. Tercero, pasó por alto que los países primero deben desarrollarse para diversificar su producción y su oferta exportable. 
Con respecto al beneficio de las exportaciones frente a las importaciones, es necesario que los productos que ofrece el país sean competitivos (Stellian y Danna-B., 2017a). Si no lo son, no podrán responder a la demanda externa y las exportaciones no aumentarán. De modo que se corre el riesgo de sustituir producción nacional por un aumento de las importaciones, y no se obtendrán los beneficios anunciados del desmonte de aranceles. La liberalización no favorece automáticamente las exportaciones. Todo depende de la existencia de ventajas comparativas, es decir, de la existencia de competitividad (Awokuse, 2008).

La idea de que el crecimiento económico sostenido resuelve la pobreza, la desigualdad y el desempleo es un consenso entre la corriente principal. Pero sostener que el crecimiento resuelve automáticamente esos problemas es contraevidente, pues la experiencia internacional ha invalidado la premisa de que el crecimiento produce un "goteo" que resuelve esos problemas. Para que el crecimiento reduzca dichos problemas, se deben cumplir varias condiciones, y el papel del Estado es esencial (Burki y Perry, 1998; Páez, Jiménez y Danna-B., 2017). Así como la liberalización no favorece automáticamente el crecimiento, tampoco reduce esos flagelos.

Aunque es posible diversificar la oferta exportable, no se logra inmediatamente. Los países en desarrollo tienen ventajas comparativas en pocos productos e inician el proceso de inserción con una oferta exportable reducida. Solo "a medida que un país se desarrolla se torna cada vez más capaz de producir una variedad creciente de productos y puede comenzar a competir con ellos en los mercados internacionales" (Agosin, 2009, p. 119). Por ello, solo diversifican su producción y sus exportaciones a medida que se desarrollan, de modo que la liberalización no favorece automáticamente dicha diversificación.

Años después de haberse impuesto la liberalización empiezan a cobrar fuerza las críticas por su incapacidad para lograr sus objetivos. Incluso quienes abogaron por ella empiezan a criticarla ${ }^{1}$. No obstante, siempre se le han hecho críticas, centradas en dos aspectos: uno sobre el costo de perseguir el crecimiento como objetivo de política económica sin reducir la pobreza y la desigualdad; otro, por apostar a políticas que pretenden un mayor crecimiento sin lograrlo.

Los fracasos de esa política y de sus maneras de implementarla no se pueden pasar por alto, menos cuando ocultan problemas que

${ }^{1}$ Ver Ostry, Loungani y Furceri (2016) y la entrevista de Maury Obstfeld, Economista Jefe del FMI [www.imf.org/external/pubs/ft/fandd/spa/2016/06/ pdf/ostry.pdf].

Revista de Economía Institucional, vol. 2 i, N.o 4i, Segundo semestre/2oi9, Pp. 7i-97 ISSN OI 24-5996/E-ISSN 2346-2450 
paga la sociedad: más impuestos, mayores costos de seguridad social y menos beneficios en salud y pensiones. Estos costos implican un mal uso de los recursos, que luego se deben reponer, de allí los programas de ajuste para equilibrar los desbalances. La política está fallando, y en vez de ponerla en discusión y diseñar una que logre los objetivos, se hacen ligeros ajustes sin modificarla.

Ya hace tiempo, Guillermo Maya (1992) argumentó que la economía colombiana sufría un enclaustramiento desde la aplicación del modelo ISI, que luego fue acentuado por la apertura. Sostenía que en los años treinta la necesidad llevó a aplicar el ISI para "sustituir progresivamente los bienes manufacturados importables por bienes nacionales, básicamente bienes de consumo final, primero, y bienes de consumo durables, después" (p. 47). En los años cincuenta se profundizó la aplicación de ese modelo y se convirtió en una política consciente. Pero en su periodo de mayor prominencia (1965-1973), se asoció con la política de promoción de exportaciones sin haber profundizado la importación de bienes intermedios y de capital. Maya se basa en ese hecho para afirmar que "con el [ISI] [...] la economía colombiana experimentó una decreciente importancia del comercio internacional, y la economía nacional sufrió un enclaustramiento, tanto físico como económico, del resto del mundo" (p. 48), del cual parece no haber salido.

Queda planteado el propósito central del artículo: discutir el modelo económico colombiano y los resultados de la liberalización comercial, para arrojar luz sobre los responsables y las ataduras que impiden liberarse de esta trampa; con ayuda de la teoría de los ciclos políticos de los negocios (CPN).

\section{DECISIONES POLÍTICAS Y COMPORTAMIENTO ECONÓMICO}

El análisis de la relación entre las decisiones políticas y su incidencia en el comportamiento de la economía ha llevado a elaborar modelos formales de ciclos políticos de los negocios (CPN). Estos modelos ayudan a entender el comportamiento de los gobernantes y de los votantes en periodos preelectorales, electorales y poselectorales.

En los estudios de esta relación se identifican dos fases: la primera, años setenta, está asociada a los trabajos de Nordhaus (1975), uno de los pioneros en el tema, de Lindbeck (1976), MacRae (1977) y Hibbs (1977). Y la segunda, años ochenta, asociada a los trabajos de Cukierman y Meltzer (1986), Rogoff y Sibert (1986), Alesina (1987), Persson y Tabellini (1990), Rogoff (1990), Alesina, Roubini y Cohen (1993). 
En la primera se usaban modelos que permitían a los gobiernos influir los resultados macroeconómicos, con motivaciones oportunistas o partidistas. Nordhaus (1975), Lindbeck (1976) y MacRae (1977) señalan que en el enfoque oportunista los gobernantes no tienen preferencias sobre políticas económicas (monetarias, cambiarias y fiscales). Por ello, eligen las que den mejores resultados macroeconómicos para obtener más apoyo electoral (Larraín y Assael, 1997). Así, en el periodo preelectoral impulsan expansiones para estimular la economía y después de ser elegidos aplican políticas contraccionistas (Escobar, 1994). Con respecto al enfoque partidista, Hibbs (1977) indica que las motivaciones de los gobernantes se derivan de la ideología de su partido, la cual orienta sus decisiones de política económica. Aunque estos desean obtener el poder, no valoran igual las expectativas de los votantes ni tienen sus mismos intereses en aspectos como el desempleo, la inflación y el crecimiento económico.

Luego de los primeros trabajos formales de los años setenta, basados en expectativas adaptativas, a finales de los ochenta aparecieron nuevos modelos, basados en expectativas racionales, que incluían aspectos de las teorías de la información y de juegos. Cukierman y Meltzer (1986), Rogoff y Sibert (1986), Rogoff (1990) y Persson y Tabellini (1990) incluyeron expectativas racionales en el enfoque oportunista, y Alesina (1987) en el enfoque partidista.

La premisa común de estos autores es que los gobernantes y los partidos compiten por el poder. Para mantenerlo, los candidatos participan en un proceso electoral en el que deben convencer a los votantes, y son tentados a manipular la política económica para mostrar mejores resultados y ser elegidos; ellos o su partido. Los demás candidatos hacen promesas y critican al gobierno para marcar diferencias en aspectos que los votantes valorarán más. Esta es la base de su hipótesis básica: los diseñadores de política recurren a la política económica antes de las elecciones para enviar señales positivas sobre variables de interés de los votantes, sabiendo que luego de ser elegidos tendrán que corregir los excesos cometidos. Los CPN permiten entonces entender el comportamiento y las motivaciones de "los políticos para permanecer en el poder, [y] el comportamiento de los votantes al momento de votar" (Coronel, 2015, p. 9).

Esos trabajos y sus modelos no habrían sido posibles sin aportes teóricos previos. Por ello reconocen las contribuciones de: Hotelling (1929), Schumpeter (1942), Buchanan (1954), Tinbergen (1956), Downs (1957), Kramer (1971) y Tufte (1978), quienes expusieron ideas sobre el comportamiento de los gobernantes, los partidos y los 
votantes durante la contienda electoral. Por ejemplo, Hotelling (1929), que aportó a la comprensión de la lucha partidista en una democracia, señaló que los programas de gobierno de los partidos tienden a coincidir porque casi nunca insisten en sus diferencias ideológicas, y hacen propuestas genéricas para solucionar problemas del votante medio (Moreno, 2013). Downs (1957) elaboró un cuerpo teórico sobre la racionalidad política y propuso discutir el comportamiento racional y las motivaciones de los gobernantes para maximizar el apoyo político. Además, argumentó que los políticos son oportunistas porque hacen sus propuestas para conseguir votos y no para implementar políticas bien definidas. Schumpeter (1942) comparó el mercado, la competencia y los consumidores con la democracia, los políticos y los votantes, lo cual sirvió de plataforma para el posterior análisis de los CPN. Él concibió la democracia como un conjunto de instituciones que cumplen una función similar a la del mercado, pues los gobernantes compiten por obtener el mayor número de votos de los consumidores políticos -los electores- (Cansino, 2008). Y Tinbergen (1956) abrió la discusión sobre la posibilidad de manipular instrumentos, fines y medios, al señalar que los diseñadores de políticas definen los objetivos de política económica.

Bajo estas consideraciones, el estudio de los CPN en la disciplina económica se ha centrado en el comportamiento de los gobernantes y votantes basado en el vínculo político-electoral, incluyendo aspectos monetarios y fiscales para mejorar el contexto de los modelos. Estos estudios también han elaborado herramientas para entender el fenómeno del poder. Por lo que sabemos, no existen trabajos sobre la relación entre los CPN y la liberalización comercial en Colombia. Los estudios disponibles se centran en la relación entre gasto público $\mathrm{y}$ periodos electorales, $\mathrm{y}$ hacen énfasis en el comportamiento de los votantes y la dinámica del gasto (Eslava, 2006). Otros autores examinan los efectos de las elecciones en el gasto de inversión en el periodo preelectoral (Drazen y Eslava, 2003). Algunos trabajos evalúan la conducta fiscal de los gobernantes municipales en la coyuntura electoral (Rubiano, 2011).Y otros analizan las etapas del proceso presupuestal, los actores de cada una de ellas y sus motivaciones (Cárdenas, Mejía y Olivera, 2006).

Algunos trabajos usan modelos para identificar los CPN en la economía colombiana durante los periodos 1935-1994 (Escobar, 1994), 1970-2005 (López y Rodríguez, 2008) y 1950-2014 (Coronel, 2015), pero no examinan la relación entre los CPN y la evolución del modelo de apertura. Otros estudian la política exterior colombiana 
desde una perspectiva económica, pero no toman en cuenta los CPN para sacar conclusiones (Ramírez, 2011; Vargas, Sosa y Rodríguez, 2012). En suma, no hay estudios que discutan el modelo económico colombiano, considerando los resultados de la liberalización comercial, a partir de la teoría de los CPN, y que identifiquen a los responsables y las ataduras que amarran la economía a la trampa de desarrollo.

Este artículo es un aporte al estudio de esa relación. Parte de dos interrogantes: ¿Cuál ha sido la influencia de los periodos presidenciales de 1990 a 2018 en la liberalización y el crecimiento económico? $¿$ ¿Hay una propensión (des)favorable hacia esas variables dependiendo del partido político del diseñador de políticas? La siguiente sección analiza el contexto político entre 1952 y 2018 destacando los periodos presidenciales posteriores a la apertura.

\section{DISEÑADORES DE POLÍTICA, LIBERALIZACIÓN Y PRODUCCIÓN}

La Constitución de 1991 marcó un antes y un después en el contexto político, pues reformó el escenario político-electoral para desmantelar el modelo bipartidista (Arboleda, 2015). Así, el sistema se abrió a nuevos competidores y candidatos independientes (Battle y Puyana, 2013) e hizo posible la participación de otros partidos.

Cuadro 1

Presidentes, partidos, periodos presidenciales y PIB, 1952-2016

\begin{tabular}{llccc}
\hline Presidentes & Partido político & $\begin{array}{c}\text { Año de } \\
\text { elecciones }\end{array}$ & $\begin{array}{c}\text { Periodo } \\
\text { presidencial }\end{array}$ & $\begin{array}{c}\text { Crecimiento } \\
\text { promedio PIB (\%) }\end{array}$ \\
\hline Gustavo Rojas Pinilla & Gobierno militar & N/A & $1952-1956$ & 5,2 \\
Junta Militar & Gobierno militar & N/A & $1956-1958$ & 2,3 \\
Alberto Lleras & Liberal & 1958 & $1958-1962$ & 5,5 \\
Guillermo León & Conservador & 1962 & $1962-1966$ & 4,6 \\
Carlos Lleras & Liberal & 1966 & $1966-1970$ & 5,9 \\
Misael Pastrana & Conservador & 1970 & $1970-1974$ & 6,5 \\
Alfonso López & Liberal & 1974 & $1974-1978$ & 4,9 \\
Julio César Turbay & Liberal & 1978 & $1978-1982$ & 3,2 \\
Belisario Betancur & Conservador & 1982 & $1982-1986$ & 3,5 \\
Virgilio Barco & Liberal & 1986 & $1986-1990$ & 4,3 \\
César Gaviria & Liberal & 1990 & $1990-1994$ & 4,3 \\
Ernesto Samper & Liberal & 1994 & $1994-1998$ & 2,8 \\
Andrés Pastrana & Conservador & 1998 & $1998-2002$ & 0,4 \\
Álvaro Uribe & Primero Colombia & 2002 & $2002-2006$ & 5,2 \\
Álvaro Uribe & Primero Colombia & 2006 & $2006-2010$ & 4,0 \\
Juan Manuel Santos & Partido de la U & 2010 & $2010-2014$ & 5,0 \\
Juan Manuel Santos & Partido de la U & 2014 & $2014-2018$ & 2,5 \\
\hline
\end{tabular}

Fuente: Dane, elaboración propia. 
Como muestra el cuadro 1, ese cambio no fue automático, pues solo desde 2002 llegaron al poder partidos diferentes a los tradicionales. Antes, el gobierno era dirigido mayoritariamente por los partidos liberal y conservador. Entre 1952 y comienzos de 2002, hubo 13 periodos presidenciales: 7 liberales, 4 conservadores y 2 militares. Entre 2002 y 2018 la presidencia ha sido ocupada por el partido Primero Colombia (2002-2010) y el Partido de la U (2010-2018), que pese a ser considerados nuevos, mantienen una relación con los partidos tradicionales, pues surgieron de disidencias del partido liberal.

Al vincular estos periodos presidenciales con el modelo económico adoptado en 1990, se observa que el comportamiento de las principales variables macroeconómicas está asociado con el partido político de turno. Es decir, aumentan o disminuyen según sea el partido en el poder, lo que podría estar relacionado con la ideología y las motivaciones del partido del diseñador de políticas conforme a la teoría de los CPN. A continuación se analiza la relación de los partidos y su inclinación a la liberalización comercial, al crecimiento del PIB, y otras variables de interés.

La liberalización comercial ha sido dirigida por el Partido Liberal y su disidencia, pues el Partido Conservador solo ha tenido un periodo de gobierno (1998-2002) desde que se inició la apertura. Esta primera deducción concuerda con los fundamentos de la teoría de los CPN, que indican que los partidos liberales son más propensos a la liberalización y los conservadores a la protección. Pero eso no se verifica en Colombia ni explica los resultados electorales, pues en las elecciones presidenciales el debate sobre la liberalización no ha sido un tema fundamental, como lo han sido la paz y el conflicto armado. El libre comercio no es producto de un debate nacional, fue una apuesta del gobierno de Virgilio Barco a finales de 1980, luego plasmada en la Constitución y después ejecutada y defendida por los gobiernos siguientes. Por tanto, no hay "diferencias entre liberales y conservadores en cuanto [...] al grado o nivel de apertura económica del país”(López y Rodríguez, 2008, p. 149).

Cuadro 2

PIB, inflación y tasa de desempleo por partido de gobierno, 1952-2016 (Porcentaje)

\begin{tabular}{lccc}
\hline Gobiernos & $\begin{array}{c}\text { Crecimiento promedio } \\
\text { del PIB }\end{array}$ & $\begin{array}{c}\text { Tasa de desempleo } \\
\text { promedio }\end{array}$ & IPC promedio \\
\hline Militares & 3,8 & N.D. & 0,10 \\
Liberales & 4,4 & 10,75 & 0,19 \\
Conservadores & 4,2 & 16,22 & 0,16 \\
Otros partidos & 3,8 & 11,51 & 0,05 \\
\hline
\end{tabular}

Fuente: Banco de la República y Dane, elaboración propia. 
El crecimiento promedio del PIB ha sido mayor durante los gobiernos liberales $(4,4 \%)$ seguido de los nuevos partidos $(4,2 \%)$. Los menores niveles de crecimiento se han registrado durante gobiernos conservadores $(3,8 \%)$ (cuadro 2). Esta segunda deducción también guardaría relación con la teoría de los CPN, según la cual los liberales dan más importancia al crecimiento que los conservadores. No obstante, aunque puede haber una relación entre los periodos presidenciales y el crecimiento del PIB, su evolución depende de otros factores externos, y no solo del diseñador de políticas.

Se pueden hacer razonamientos similares con respecto a otras variables. Sobre el desempleo y la inflación se podría decir que el menor nivel de desempleo promedio ha ocurrido durante gobiernos liberales $(10,75 \%)$ seguido de los nuevos partidos (11,63\%). En cambio, durante los gobiernos conservadores fue del 16,22\%. Asimismo, en los gobiernos liberales el nivel promedio de inflación ha sido mayor que en los gobiernos conservadores, $0,19 \%$ y $0,16 \%$ respectivamente (cuadro 2). Estas deducciones también guardarían relación con la teoría de los CPN, que señala que los liberales son más afines a un bajo desempleo y los conservadores a una inflación baja (Hibbs, 1977). Sin embargo, al generalizar el comportamiento promedio de la economía durante el conjunto de periodos presidenciales por partido "es necesario tener en cuenta el hecho de que a los cambios de gobierno se les puede estar imputando otros shocks exógenos de naturaleza distinta que también afectaron a la economía" (Escobar, 1994, p. 28).

Grosso-modo, se observa una propensión de los gobiernos liberales a estimular el crecimiento y reducir el desempleo, puesto que el crecimiento promedio del PIB ha sido mayor en los gobiernos de ese partido y la tasa de desempleo menor. Se había considerado a priori que los diseñadores de políticas liberales también habrían promovido la liberalización comercial del país. Pero no es posible concluir que los liberales sean propensos a la apertura porque la liberalización fue una apuesta de gobierno, que data de 1986, y no un tema de debate electoral. El principal objetivo de los conservadores ha sido mantener una inflación baja, aunque haya implicado menor crecimiento y mayor desempleo.

Aún falta responder uno de los interrogantes: dar luces sobre las ataduras que sostienen la trampa de desarrollo. La sección 4 analiza qué cambió con el modelo de apertura en cuanto al comportamiento de algunas variables macroeconómicas. 


\section{¿QUÉ CAMBIÓ CON LA LIBERALIZACIÓN COMERCIAL?}

Los resultados se presentan en dos partes. Primero se valora el impacto de la liberalización comercial en el crecimiento y el desarrollo. Y luego los efectos sobre la diversificación de las exportaciones y la dependencia de las exportaciones de bienes básicos y recursos naturales.

\section{CRECIMIENTO Y DESARROLLO EN EL MARCO DE LA LIBERALIZACIÓN}

La liberalización comercial colombiana pretendía lograr un mayor crecimiento suponiendo que la eliminación de los obstáculos al comercio dinamizaría las exportaciones y daría lugar a un crecimiento jalonado por las exportaciones. Partió del siguiente antecedente:

La economía colombiana ha logrado importantes avances en términos de crecimiento del producto y del ingreso nacional, en un marco de estabilidad con niveles de inflación controlados y un notable crecimiento y diversificación de las exportaciones, gracias a las cuales las condiciones cambiarias y de balanza de pagos han podido tener un alto grado de solidez. Todos estos logros se encuentran vinculados con un modelo de desarrollo que combina una estrategia de sustitución de importaciones y protección a la industria nacional, con una estrategia de promoción de exportaciones (Conpes, 1990, p. 4).

Urrutia y Posada (2007) subrayan que en el siglo XX Colombia tuvo un crecimiento económico estable y redujo la brecha de ingreso per cápita con respecto a Europa y al Este de Asia pero no frente a Estados Unidos. Con respecto a este último país, señalan que para lograrlo habría sido necesario aumentar la productividad total de los factores, lo que asocian a la capacidad para adoptar cambios técnicos que se adquiere "en una sociedad muy abierta"; contrarrestar la violencia y modificar la percepción de la sociedad ideal futura. Y argumentan que la economía podría crecer más en este siglo si se cambiaran algunas de las políticas aplicadas en el siglo pasado.

La idea de contrarrestar la violencia y modificar el ideal de la sociedad futura es novedosa en los trabajos sobre determinantes del crecimiento, pero no en los estudios de los determinantes del desarrollo, que difiere del crecimiento. Con esta óptica, Silva Colmenares (2010) indica que el crecimiento está asociado a la producción de bienes y servicios, y que el desarrollo consiste en el mejoramiento de las condiciones de vida de las personas, satisfaciendo sus necesidades. Así, “el 'crecimiento' es 'económico' [y] el 'desarrollo' es 'humano”' (ibíd., p. 89). De modo que el crecimiento es el medio y el desarrollo es el fin.

En Colombia parece haberse invertido la relación entre medios y fines, lo que ha llevado a buscar con más insistencia el medio, como si 
fuese un fin. Gillis, Perkins et al. (1998) subrayan que el crecimiento es una condición necesaria pero no suficiente para el desarrollo, por lo que se requieren ambos procesos, que deben complementarse. Esto contrasta con los planteamientos del Conpes (1990) sobre el modelo de desarrollo adoptado desde 1990. El Conpes no menciona la importancia del desarrollo y solo se preocupa por las futuras tasas de crecimiento. Esta omisión es aún más diciente al saber que cuando se inició el modelo de apertura, ya existían en el país planteamientos sobre la importancia de no perder de vista el desarrollo y la necesidad de unirlo al crecimiento, que no se consideraron.

Uno de los mayores consensos sobre el desempeño económico entre 1950-1990 es que los logros de la economía obedecieron al modelo de desarrollo. Sobre ese periodo el Conpes mencionado señala que "el crecimiento del PIB no [fue] negativo en ningún año; la inflación [se mantuvo] bajo control; las exportaciones [alcanzaron] un notable grado de diversificación y las condiciones cambiarias y de balanza de pagos [tuvieron] un alto grado de solidez" (p. 20). Así, deja la sensación de que el modelo anterior a la apertura estaba funcionando y que se había emprendido el camino de la diversificación exportadora.

Sin embargo, esa senda de crecimiento comenzó a ser obstaculizada a finales de 1980 luego de la crisis de la deuda y la "década perdida", cuando se enfrentaron dificultades fiscales que limitaron el desarrollo exportador. Por ello, el Conpes (1990) mencionó otro antecedente para justificar el nuevo modelo de desarrollo:

A pesar de sus éxitos, las limitaciones del modelo de crecimiento económico han empezado a ser evidentes [...] Se manifiestan en la tendencia a la baja de la tasa de crecimiento de la economía en el largo plazo y en el hecho de que, pese a su favorable evolución reciente, las exportaciones no tradicionales alcanzan todavía proporciones excesivamente bajas de la producción nacional (p. 4).

La preocupación en ese momento era el modelo de crecimiento, que imponía limitaciones a la tendencia de largo plazo. Según el mismo Conpes, la oferta exportable se concentraba en bienes tradicionales y era necesario diversificarla para lograr el crecimiento anhelado mediante la liberalización. Sin embargo, el régimen de crecimiento posterior a la apertura se basó en el mercado inmobiliario y no en las exportaciones como se esperaba. Se articuló alrededor de la dinámica especulativa del mercado inmobiliario impulsada por la financiación internacional, muy vulnerable a las crisis. Si faltara esa financiación, la actividad económica sería afectada necesariamente pues la dinámica especulativa corría el riesgo de romperse, anulando sus efectos de 
arrastre sobre la economía. Danna-B. (2012) señala que los capitales extranjeros de corto plazo empezaron a disminuir desde 1995, y se redujeron visiblemente en 1999. Simultáneamente, el precio de la vivienda disminuyó desde 1995 y el crédito hipotecario desde 1997. Se reunieron todos los elementos para interrumpir la dinámica. El motor de crecimiento de la economía desapareció y advino la crisis.

Así, Colombia vivió la peor crisis del siglo XX después de la apertura. Como muestra la gráfica 1, la tasa de crecimiento empezó a estancarse desde 1994-1995, descendió en 1996 y cayó al -4,2\% en 1999. Solo en 2003 superó el nivel de 1989, lo cual contrasta con el crecimiento alcanzado en la "década perdida", durante la cual la tasa de crecimiento fue siempre positiva, del 5,8\% en 1986 y del 5,4\% en 1987. Si se compara el crecimiento promedio del periodo 1951-1989 (4,7\%) con el de 1990-2016 (3,6\%), es claro que la liberalización del comercio no logró lo que se pretendía; alcanzar tasas de crecimiento superiores a las del periodo pre-apertura y seguir una senda de crecimiento estable ${ }^{2}$.

Gráfica 1

Tasa de crecimiento del PIB, 1950-2016

(Porcentaje)

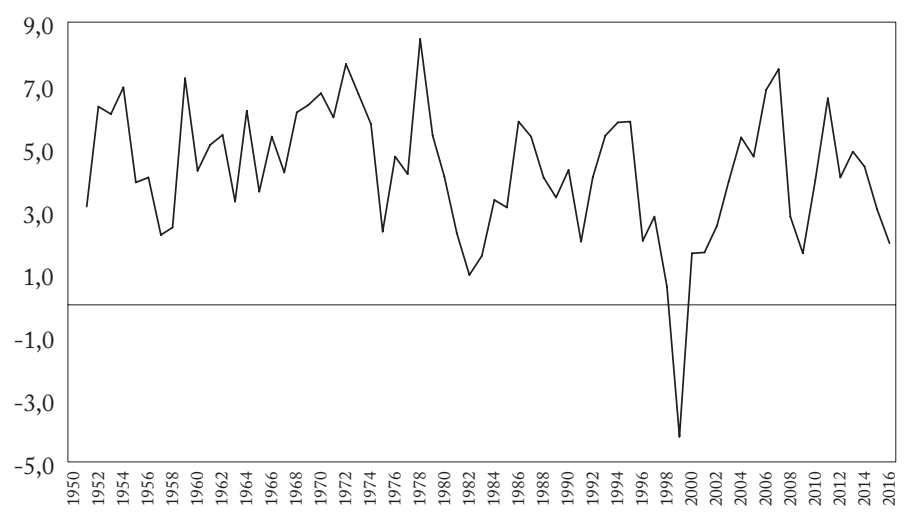

Calculada a precios de 2005.

Fuente: Dane y Banco de la República, elaboración propia.

Esta crisis, denominada “crisis de la transición" (ibíd., p. 122), afectó las condiciones de vida de los colombianos, de modo que la apertura tampoco logró el crecimiento que debía solucionar los problemas de

${ }^{2}$ E1 crecimiento promedio del 4,7\% durante 1951-1989 se logró pese a las crisis del petróleo (1975) y de la deuda (1982). 
la economía colombiana. Por el contrario, dio origen a la crisis que empeoró dichos problemas:

- En 1990 los ingresos del décimo decil eran 21,2 veces mayores que los del primer decil; en 1999 ya eran 40,6 veces mayores ${ }^{3}$.

- El coeficiente de Gini pasó de 0,51 en 1990 a 0,59 en $1998^{4}$. Y según ese mismo índice se amplió la brecha entre áreas urbanas y rurales (Forero y Ezpeleta, 2007).

- Entre 1995 y 1999 la brecha de pobreza pasó del 29\% al 34\%, la tasa de pobreza pasó del $60 \%$ al $64 \%$ y la de pobreza extrema del 21\% al 23\% (World Bank, 2002).

- Durante 1985-1989 la tasa de desempleo tuvo una tendencia decreciente. Con la apertura, esa tendencia se mantuvo hasta 1995, con tasas de un dígito, pero empezó a aumentar en 1996 (11,20\%), y se elevó al 15,23\%, al 19,38\% y al 20,22\% en 1998 , 1999 y 2000 respectivamente ${ }^{5}$.

A esto se suma el hecho de que tampoco se diversificó la oferta exportable y se acentuó la dependencia con respecto a las actividades primarias, extractivas y de bienes básicos.

\section{OFERTA EXPORTABLE, DEPENDENCIA DE LOS BIENES}

\section{BÁSICOS Y ESTRUCTURA PRODUCTIVA}

Históricamente, Colombia se ha especializado en "exportaciones tradicionales", término que designa las exportaciones de café, petróleo, carbón, esmeraldas, banano, níquel y oro. Lamentablemente, los precios de estos productos fluctúan en los mercados internacionales, lo que afecta el crecimiento del PIB y los ciclos económicos mediante diferentes canales: reducción de los términos de intercambio y del ingreso nacional, deterioro de la balanza comercial, descenso del capital extranjero y depreciación de la moneda (Banco de la República, 2017). Por ello, con la apertura se esperaba encontrar nuevos mercados que aumentaran la participación de las exportaciones no tradicionales frente a las exportaciones tradicionales en el total ${ }^{6}$.

${ }^{3}$ [https://data.worldbank.org/indicator].

${ }^{4}$ [https://datos.bancomundial.org/indicador/SI.POV.GINI].

${ }^{5}$ [www.banrep.gov.co/es/tasas-empleo-desempleo].

${ }^{6}$ Exportaciones no tradicionales: sector agropecuario (algodón, arroz, flores, tabaco, carne de res, frutas y legumbres), sector industrial (alimentos y bebidas -incluye azúcar-, hilados y tejidos, confecciones, productos de plástico y caucho, cuero y sus manufacturas, madera y sus manufacturas, artes gráficas y editoriales, industria química, minerales no metálicos, metales comunes, maquinaria y equipo, y material de transporte) y sector minero (fueloil y derivados, carbón y esmeraldas) (Greco, 2002). 
La economía colombiana ha tenido grandes dificultades para diversificar las exportaciones, antes y después de la liberalización comercial. Pero, ¿qué ha impedido diversificarlas? Sobre los años anteriores a la apertura, Greco (2002) indica que "en el siglo XX solo en los periodos comprendidos entre 1908-1921 y 1965-1999 la participación de las exportaciones diferentes a café, petróleo crudo, banano y oro sobrepasó el 15\%" (p. 8). Su justificación de lo que ocurrió entre 1908 y 1921 es que las exportaciones se basaron en la disponibilidad de recursos naturales y el uso de mano de obra no calificada, en especial las de cuero, platino y tabaco. Esta explicación parece sugerir que la decisión de producir esos bienes y aprovechar los recursos naturales fue decisión de una mano invisible, impulsada por esos aspectos que actuaron como incentivo. No obstante, Greco no discute cómo ni quién tomó esas decisiones, y deja la sensación de que la economía por sí misma pasa de café a cuero, de cuero a banano, de banano a tabaco y de tabaco a platino. Así "invisibiliza" a los agentes y las relaciones que los vinculan, así como los intereses que los mueven.

Antes de la apertura (1950-1980), la elite política pensaba que podía industrializar el país y diversificar la oferta exportable mediante el modelo ISI y la promoción de exportaciones. Pero fue imposible sostener un alto nivel de exportaciones, lo que limitó la industrialización por la escasez de divisas que restringió las importaciones necesarias para el desarrollo industrial y diversificar la producción ${ }^{7}$. Otros factores reforzaron la escasez de divisas y sus efectos sobre la industrialización, como el aumento del servicio de la deuda externa y el deterioro de los términos de intercambio.

Entre 1970-1990, el servicio [de la deuda] aumentó progresivamente un $1253 \%$, mientras que los términos de intercambio no presentaron la misma progresión, comportándose de manera caótica a corto plazo y registrando una tendencia a permanecer en el mismo nivel a largo plazo [...] En estas condiciones, era imposible utilizar de manera sistemática y sostenida las divisas generadas por las exportaciones para financiar las importaciones de bienes de alto contenido tecnológico. Si bien el Estado intentó favorecer las exportaciones agrícolas, mineras y de hidrocarburos para disponer de las divisas en cuestión [...], el servicio de la deuda hacía que esas medidas fueran insuficientes (Danna-B., 2012, p. 35).

Al revisar la participación de las exportaciones tradicionales y no tradicionales en el total se observa que la apertura no ha contribuido

7 Entre 1950 y 1989, Colombia exportó café (60,2\%) y demás bienes agropecuarios: banano, flores, carne, azúcar y tabaco (5,9\%); minería: petróleo, oro, carbón $(11,5 \%)$, y otros productos: incluidas las manufacturas $(22,3 \%)$ (Villar y Esguerra, 2005). 
a la diversificación. Paradójicamente, ha llevado a una mayor exportación de productos tradicionales y a la primarización de la economía. Como muestra la gráfica 2, después de la apertura las exportaciones tradicionales representan la mitad, e incluso un poco más, de las exportaciones totales y parecen reflejar una resistencia a bajar ese límite, lo cual sugiere que la economía está anclada en esos productos tradicionales, a pesar de la liberalización de los mercados y aunque la apertura prometió modificar esta relación.

Gráfica 2

Exportaciones tradicionales y no tradicionales, 1974-2016

(Porcentaje)

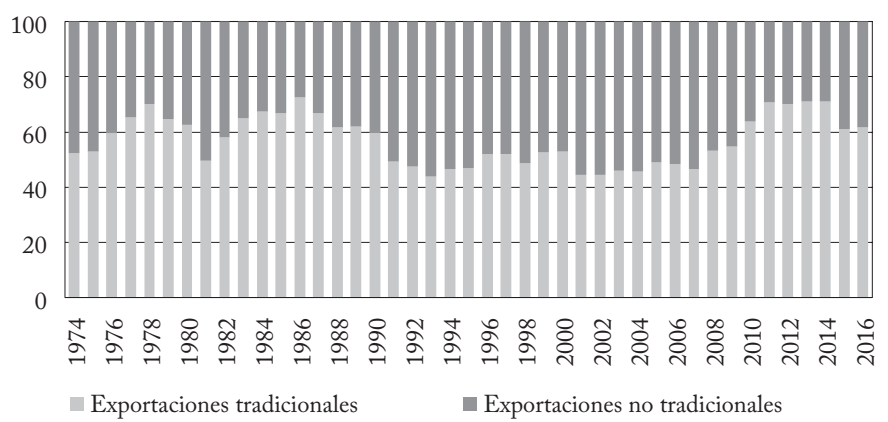

Fuente: Dane y cálculos DNP-DDE, elaboración propia.

Por otra parte, en el periodo 1974-1989 las exportaciones tradicionales representaron en promedio el 62\% y en el periodo 1990-2016 el 54\%. Esta leve disminución puede indicar cierta diversificación, pero no significa que continúe ni que sea sostenible, y menos aún que se oriente en favor de las exportaciones de productos industriales. Aunque con la liberalización del comercio se pretendía modificar la estructura de las exportaciones sigue persistiendo el predominio de los bienes básicos ${ }^{8}$.

Y no solo eso; las exportaciones de productos tradicionales se han acentuado. La gráfica 3 muestra tres hechos destacables: 1) la participación del sector industrial en las exportaciones totales viene disminuyendo; 2) la participación del sector minero tuvo un fuerte aumento en 2007-2014, periodo de bonanza minero-energética que vivió el país por los buenos precios internacionales de algunos bienes

${ }^{8}$ Entre 1995 y 2016 el Índice Herfindahl-Hirschmann (IHH) ha sido superior a 0,18 , lo que indica que las exportaciones están concentradas en pocos productos. Si bien, entre 2003 y 2007 el IHH disminuyó, no se podría asegurar que se tienda a la diversificación, pues entre 2010 y 2015 registró los valores máximos de concentración de todo el periodo de estudio. 
primarios; y 3) las exportaciones del sector agropecuario tienden a disminuir. En suma, hay un cambio reciente en la participación sectorial, de la industria (exportaciones no tradicionales) a la minería y un descenso del sector agrícola. Esto confirma la conclusión de Restrepo (2001): "aunque las exportaciones no tradicionales han ganado participación dentro de las exportaciones totales [...], Colombia no tiene una estructura productiva para participar con éxito en la dinámica de la demanda mundial" (p. 150).

Gráfica 3

Participación sectorial en las exportaciones, 1995-2017 (Porcentaje)

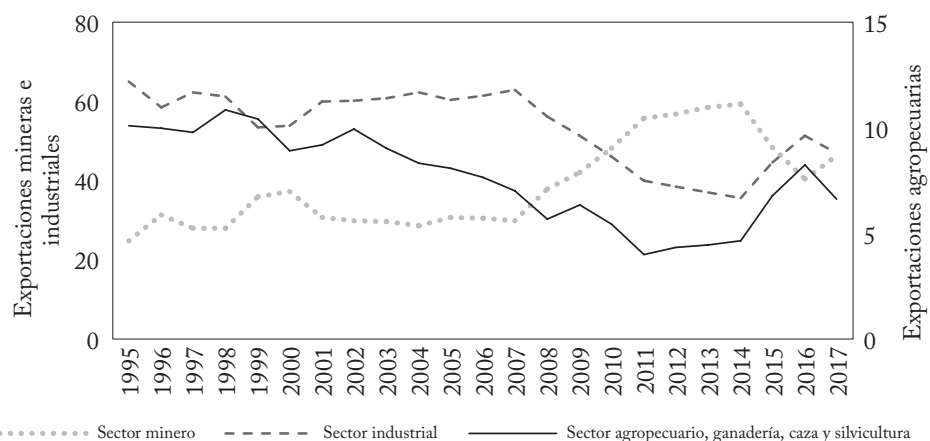

Fuente: Dian y Dane, elaboración propia.

Cuestionar sin matices el nivel de exportaciones tradicionales de un país puede ser equívoco, pues puede estar aprovechando sus ventajas comparativas, que en el caso colombiano se podrían asociar a la producción agrícola y los recursos naturales no renovables ${ }^{9}$. Sería entonces razonable que un país exporte los excedentes de bienes en los que tiene ventajas comparativas y además satisfaga la demanda interna. Pero los datos de Colombia no indican que suceda así. Al contrario, concuerdan con el trabajo de Greco (2002), donde se muestran los periodos en que las exportaciones diferentes de café, petróleo crudo, banano y oro estuvieron determinadas por la disponibilidad de recursos naturales. Hoy la historia se repite, con un agravante: desde 2007 la economía empezó a depender de las exportaciones mineras.

De acuerdo con Bonilla, durante los últimos 20 años se consolidó en Colombia un modelo de desarrollo dependiente de las exportaciones mineras y de hidrocarburos, lo que "supone un regreso al predominio

9 Para las ventajas comparativas de Colombia, ver Danna (2012, 2017), Stellian y Danna (2017b), Danna, Stellian, Mercado y Páez (2017). 
del sector primario en detrimento de la industria, con el supuesto de que estamos sentados sobre 'una mina de oro' compuesta por hidrocarburos y minerales” (2011, p. 46). Misas, Ramírez y Silva (2001) sostienen que la demanda externa tiene un papel preponderante en la exportación de bienes no tradicionales, así como los precios relativos. Y como se ha mostrado en este artículo, la demanda externa también jugaría un papel preponderante en la exportación de bienes tradicionales, lo que lleva a plantear que la economía colombiana está atrapada en un modelo de desarrollo que le impide diversificar sus exportaciones.

Otro hecho que muestra la dependencia de la economía colombiana con respecto al sector externo y a los bienes básicos es la participación sectorial en las exportaciones y las importaciones. La apertura no ha servido para explotar las ventajas comparativas. Al contrario, ha creado desventajas que lesionan la estructura productiva y pueden ser una explicación de la trampa que señalamos. Si el país tiene recursos naturales abundantes y tierras con distintos pisos térmicos para cultivar diversos productos agrícolas, sería razonable que aprovechara esas ventajas. Para poder hacerlo y mejorar los niveles de productividad y de competitividad, aún es necesario que se introduzcan grandes cambios técnicos, lo que se pretendió hacer con el modelo ISI y no se consiguió.

Gráfica 4

Participación de los bienes importados, 1974-2016 (Porcentaje)

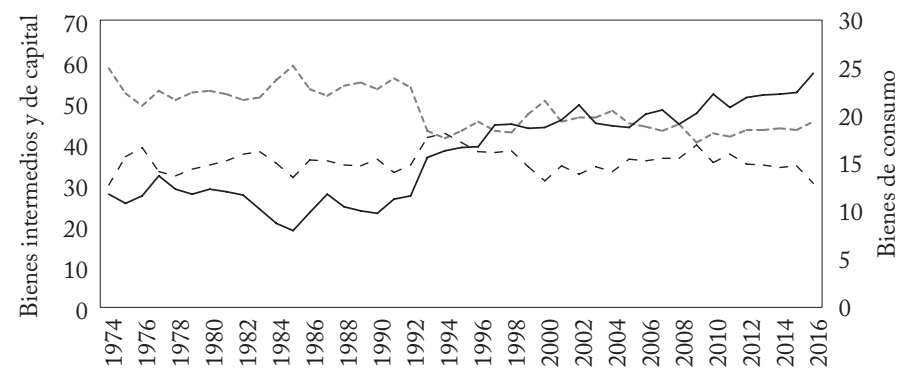

--_--- Bienes intermedios _ - - - Bienes de capital _ Bienes de consumo

Fuente: DIAN y cálculos DNP-DDE; elaboración propia.

La gráfica 4 muestra que entre 1974 y 1989 la importación de bienes intermedios representaba en promedio el $53 \%$ y la de bienes de capital el 35\%, mientras que los bienes de consumo solo representaban el 
$11 \%$. Esto es coherente con el desarrollo basado en la explotación de las ventajas y con el intento de tecnificar los procesos productivos para lograr la industrialización. En cambio, en contra de los argumentos que llevaron a adoptar la política de apertura y de los objetivos que supuestamente cumpliría, los resultados observados son adversos, tanto porque se ha perdido mucho de lo que se había construido, como porque no se ha cumplido lo que se prometió. Los datos son elocuentes: después de la liberalización comercial (1990-2016), la importación de bienes intermedios se redujo a un nivel promedio del $45 \%$ y la de bienes de capital se han mantenido en el 35\% en promedio. El costo de la apertura se ha pagado en bienes de consumo, que hoy representan un $25 \%$.

Esto lleva a pensar que el modelo de desarrollo y la liberalización comercial han sido un fracaso considerando el potencial de la economía colombiana, pues luego de la apertura, las profundas reformas institucionales y el costo fiscal, el país importa cada vez más bienes de consumo, exporta minerales y productos tradicionales, y no ha podido industrializar la economía y diversificar las exportaciones.

Hay otros factores que contribuyendo a esa situación. La caída de los precios del petróleo en la segunda mitad de 2014 deterioró los términos de intercambio e hizo necesario ajustar la política económica para evitar el colapso de la economía (gráfica 5). Eso fue necesario, aunque el régimen cambiario flexible del país puede absorber choques externos y detener la caída pronunciada del crecimiento (Banco de la República, 2017).

\section{Gráfica 5}

Términos de intercambio de Colombia (1995-2017)

Base: $2000=100$

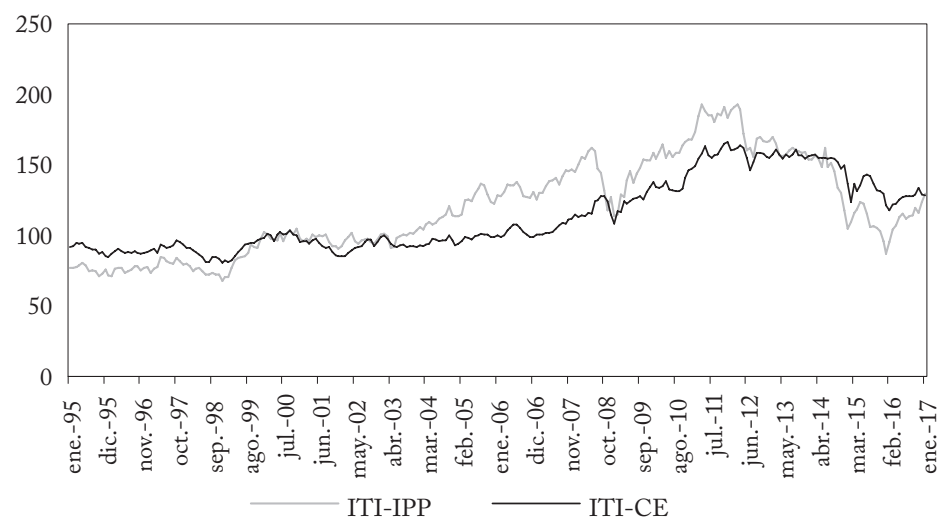

Fuente: Banco de la República, elaboración propia. 
E1 deterioro de los términos de intercambio ha restringido aún más la modernización de la economía, que también ha sido limitada por la baja productividad de los factores, lo que ha afectado el crecimiento. E1 Conpes (2016) plantea que el crecimiento económico de largo plazo se basa en aumentos de la productividad, y reconoce que una de las causas de la baja productividad es el escaso número de actividades y productos competitivos. Por ello, señala que Colombia tiene un gran rezago en materia de productividad, pues "la [Productividad total de los factores] tuvo un crecimiento anual nulo o negativo durante 12 años en el periodo 1991-2015" (p. 34), y este estancamiento es responsable de la reducción de 0,5 puntos porcentuales en el crecimiento del PIB. Otra razón para hablar de la trampa en que está atrapada la economía es la incapacidad para mejorar la productividad multifactorial, lo cual es más grave cuando el mismo Conpes reconoce que el sector de servicios es el que más ha contribuido a aumentarla; mientras que la contribución de la industria, que debería hacer grandes aportes, ha sido apenas de un $0,1 \%$.

\section{CONCLUSIÓN}

La modernización de una economía está asociada a su modelo de desarrollo; así, no solo depende de la acumulación de factores y de su productividad en el largo plazo, sino también de las políticas adoptadas para ello. Sin embargo, el interés por elevar el nivel de modernización e implementar políticas de desarrollo está sujeto a las valoraciones de los gobernantes, que los llevan a identificar ciertas actividades económicas para asignar presupuesto y orientar la política de gastos e impuestos, que es diseñada por especialistas y asesores. Así, ellos actúan como delegatarios y no de manera autónoma. Cabe destacar este hecho, pues la delegación no es una relación de subordinación entre el gobernante y los diseñadores de política. Detrás del gobernante están quienes financiaron su campaña y esperan obtener rentas. Así, las políticas que terminan diseñando los especialistas (agentes) son dictadas por los financiadores (principal) y no por el gobernante, quien en muchos casos se distancia de su ideología y abandona las promesas de la campaña electoral. De modo que según sean los intereses de los agentes así serán las políticas de desarrollo, que por lo general están subordinados a los intereses del capital.

Por ello, la falta de modernización obedece al modelo adoptado hace un cuarto de siglo y a las políticas asociadas, que mantienen el país en una trampa de desarrollo que impide tecnificar la estructura 
productiva, diversificar la oferta exportable, mejorar la productividad, reducir la dependencia de bienes básicos y avanzar por una senda de crecimiento estable y positiva. Este artículo muestra que el modelo de apertura, impuesto con el argumento de que modernizaría la economía y mejoraría la tasa de crecimiento, no ha logrado ni lo uno ni lo otro. En cambio, la economía se ha orientado a producir bienes primarios de bajo valor agregado para la exportación; además, la tasa de crecimiento ha sido menor y más volátil por su dependencia de bienes básicos y productos mineros.

La evidencia que se ha presentado muestra que la economía colombiana no se ha diversificado y que su estructura productiva no se ha modernizado, pese al modelo de desarrollo que tenía esos propósitos. Esta es una razón para que el debate y la discusión pasen del ajuste sistemático de las políticas a una reflexión más profunda sobre el modelo, pues reconocer el fracaso del modelo es reconocer que la dirigencia política, asociada a cierta élite empresarial, también ha fracasado, y que no tiene sentido mantener sus propuestas con ligeras alteraciones. Es más conveniente modificar la actual orientación de la economía y corregir las fallas que impiden aprovechar su potencial.

\section{REFERENCIAS BIBLIOGRÁFICAS}

Agosin, M. (2009). Crecimiento y diversificación de exportaciones en economías emergentes. Revista de la Cepal, 97, 115-131.

Alesina, A. (1987). Macroeconomic policy in two-party system as a repeated game. Quarterly Journal of Economics, 102, 651-678.

Alesina, A., Cohen, R. et al. (1993). Electoral business cycle in industrial democracies. European Journal of political Economy, 9(23), 1-25.

Arboleda, P. (2015). Los partidos políticos en la Constitución de 1991. Medellín: FUNLAM.

Awokuse, T. (2008). Trade openness and economic growth: Is growth export-led or import-led? Applied Economics, 4O(2), 161-173.

Balassa, B. (1985). Exports, policy choices and economic growth in developing countries after the 1973 oil shock. Journal of Development Economics, 18(1), 23-35.

Banco de la República. (2017). Informe de la Junta Directiva al Congreso de la República.

Battle, M. y Puyana, J. (2013). Reformas políticas y partidos en Colombia: cuando el cambio es la regla. Politai, 4(7), 73-88.

Bejarano, J. (1998). Industrialización y política económica 1950-1976. En M. Arrubla et al., Colombia hoy, 8. ${ }^{a}$ ed. Bogotá: Siglo XXI.

Berr, E. y Combarnous, F. (2004). L'impact du consensus de Washington sur les pays en développement: une évaluation empirique. Groupe d'Economie du Développement de l'Université Montesquieu Bordeaux IV.

Berr, E. (2003). La dette des pays en développement: bilan et perspectives. Revue Africaine de sciences économiques et de gestion, 5(2), 3-32. 
Bonilla, R. (2011). Apertura y reprimarización de la economía colombiana. Nueva Sociedad, 231, 46-65.

Buchanan, J. (1954). Social choice, democracy and free markets. Journal of political economy, 62(2), 114-123.

Burki, S. y Perry, W. (1998). Más allá del Consenso de Washington: la hora de la reforma institucional. Washington DC: BIRF-BM.

Cámara de Comercio de Bogotá. (1993). La apertura en Colombia: agenda de un proceso. Bogotá: Tercer Mundo.

Cansino, C. (2008). La muerte de la ciencia politica. Buenos Aires: Sudamericana.

Cárdenas, M., Mejía, C. et al. (2006). La economía política del proceso presupuestal en Colombia. Series de Estudios Económicos y Sectoriales, RE3-06-008, 2-79.

CNUCED (2003). Diversificación de las exportaciones, acceso a los mercados y competitividad.

Conpes. (1990). Programa de modernización de la economía colombiana (Conpes: DNP-2.465-J).

Conpes. (2016). Politica nacional de desarrollo productivo (Conpes: DNP$3.866)$.

Coronel, J. (2015). Ciclo politico de los negocios en Colombia 1950-2014. Tesis de maestría. Medellín: Universidad Nacional de Colombia.

Cukierman, A. y Meltzer, A. (1986). A positive theory of discretionary policy, the cost of democracy government and the benefits of constitucion. Economic Inquiry, 24(3), 367-388.

Danna-B., J. (2017). La alianza del pacífico+4 y la especialización regional de Colombia: Una aproximación desde las ventajas comparativas. Cuadernos de Administración, 30(55), 163-192.

Danna-B., J., Stellian, R. et al. (2017). Análisis retrospectivo de la integración internacional del sector manufacturero colombiano en el marco del Tratado de Libre Comercio con Estados Unidos. Bogotá: Fundación Universitaria los Libertadores.

Danna-B., J. (2012). L'intégration économique internationale de la Colombie (1990-2010): une approche en termes d'économie politique internationale. Tesis de Doctorado. Grenoble: Universidad de Grenoble.

Dingemans, A. y Ross, C. (2012). Los acuerdos de libre comercio en América Latina desde 1990. Una evaluación de la diversificación de las exportaciones. Revista Cepal, 108, 28-50.

DNP. (1991). Plan de desarrollo económico y social 1990-1994: "La revolución pacífica".

Downs, A. (1957). An economic theory of political action in a democracy. Journal of political economy, 65(2), 135-150.

Drazen, A. y Eslava, M. (2003). The political business cycle in Colombia on the National and Regional level. Archivos de economía 3616, DNP.

Echavarría, J. (2001). Colombia en la década de los noventa: neoliberalismo y reformas estructurales en el trópico. Cuadernos de economía, 20(34), 57-102.

Edwards, S. (1994). Trade and industrial policy reform in Latin America. NBER working paper 4772. 
Escobar, A. (1994). Ciclos políticos y ciclos económicos en Colombia: 1935-1994. Bogotá: Fedesarrollo.

Eslava, M. (2006). Ciclos políticos de la política fiscal con votantes opuestos al déficit. El Trimestre Económico, 73(290), 289-336.

Forero, J. y Ezpeleta, S. (2007). Las brechas entre el campo y la ciudad en Colombia 1990-2003, y propuestas para reducirlas. Bogotá: Cepal.

García, J. (2002). Liberalización, cambio estructural y crecimiento económico en Colombia. Cuadernos de Economía, 21(36), 189-244.

Gelbard, E. (1990). Dinámica de la concentración industrial y de la productividad en relación a la apertura de la economía. Desarrollo Económico, 30(117), 27-54.

Gillis, M., Perkins, D. et al. (1998). Economie du développement. Bruxelles: De Boeck Université.

Greco. (2002). El crecimiento económico colombiano en el siglo XX. Bogotá: Banco de la República.

Hibbs, D. (1977). Political parties and macroeconomic policy. The American Political Science Review, 71(4), 1467-1487.

Hotelling, H. (1929). Stability in competition. The economic journal, 39(153), 41-57.

Kramer, G. (1971). Short-term fluctuations in U.S. voting behavior 18961964. The American Political Science Review, 65(1), 131-143.

Larraín, F. y Assael, P. (1997). El ciclo político económico en Chile en el último medio siglo. Estudios Públicos, 68, 198-214.

Lindbeck, A. (1976). Stabilization policy in open economies with endogenous politicians. The American Economic Review, 68(2), 1-19.

López, M., Gallón, S. et al. (2002). E1 ciclo político-económico en Colombia 1925-1999. Lecturas de Economia, 56, 7-30.

López, M. y Rodríguez, A. (2008). Ciclos políticos y agregados macroeconómicos: Un análisis para el caso colombiano (1970-2005). Perfil de Coyuntura Económica, 12, 137-158.

López, D., López, E. et al. (2015). Colombia en el comercio mundial (1992-2012): desempeño de las exportaciones colombianas. Borradores de Economía, 885, 1-59.

Lora, E. (2001). Las reformas estructurales en América Latina: Qué se ha reformado y cómo medirlo. BID. Documento de trabajo-348.

MacRae, C. (1977). A political model of the business cycle. Journal of Political Economy, 85(2), 239-263.

Maya, G. (1992). Apertura económica y agricultura en Colombia. Ensayos de Economia, 3(5), 47-77.

McKinnon, R. (1964). Fiscal policy for growth without inflation. American Economic Review, 54(1), 183-185.

Misas, M., Ramírez, M. et al. (2001). Exportaciones no tradicionales en Colombia y sus determinantes. Ensayos sobre Politica Económica, 19(39), 73-114.

Moreno, A. (2013). Teorías y modelos del ciclo político de los negocios, Universidad Externado de Colombia, (45), 3-38.

Nordhaus, W. (1975). The political business cycle. The Review of Economic Studies, 42(2), 169-190. 
Ocampo, J. y Villar, L. (1992). Trayectoria y vicisitudes de la apertura económica colombiana. Pensamiento Iberoamericano, 21, 165-189.

OMC. (1996). Colombie: le processus de libéralisation stimule l'investissement et conduit à une plus grande intégration à l'économie mondiale, [https://www.wto.org/french/tratop_f/tpr_f/tp039_f.htm].

Ostry, J. y Loungani, P. (2016). Neoliberalismo: ¿Un espejismo? Finanzas y Desarrollo, 53(2), 38-41.

Páez, P., Jiménez, W., y Danna-B., J. (2017). Necesidades humanas y política social. Revista Republicana, 22, 131-158.

Persson, T. y Tabellini, G. (1990). Macroeconomic policy, credibility and politics. Ámsterdam: Hardwood Academic Publishers.

Ramírez, S. (2011). El giro de la política exterior colombiana. Nueva Sociedad, 231, 79-95.

Restrepo, R. (2001). La economía colombiana en la década de los noventa: dilema entre el mercado interno y el mercado internacional. Ensayos de economía, 12(18), 145-152.

Rogoff, K. (1990). Equilibrium political budget cycles. American Economic Review, 80(1), 21-36.

Rogoff, K. y Sibert, A. (1986). Elections and macroeconomic policy cycles. NBER working paper 1838.

Rostow, W. (1963). Les étapes de la croissance économique. París: Seuil.

Rubiano, N. (2011). Ciclo político de los negocios y tamaño municipal: Colombia 1989-2008. Revista de Economia Institucional, 25(13), $105-136$.

Schumpeter, J. (1942). Capitalismo, socialismo y democracia. Barcelona: Folio.

Silva-Colmenares, J. (2010). Colombia: crecimiento económico y desarrollo bumano. Bogotá: Fundación Universidad Autónoma de Colombia.

Stellian, R. y Danna-B., J. (2017a). Protocolo de medición de las ventajas comparativas de los artículos manufacturados colombianos frente a Estados Unidos. En J. Danna-B. (ed.). Análisis retrospectivo de la integración internacional del sector manufacturero colombiano en el marco del Tratado de Libre Comercio con Estados Unidos (pp. 55-66). Bogotá: Fundación Universitaria los Libertadores.

Stellian, R. y Danna-B., J. (2017b). Competitividad de los productos agropecuarios colombianos en el marco del tratado de libre comercio con los Estados Unidos: análisis de las ventajas comparativas. Revista Cepal, 122, 139-163.

Stiglitz, J. E. (2010). Le triomphe de la cupidité. Francia: Les Liens qui Libèrent.

Tinbergen, J. (1956). Economic policy: Principles and design. The Economic Journal, 69(274), 353-356.

Tufte, E. (1978). Political control of the economy. New Jersey: Princeton University Press.

Urrutia, M. y Posada, C. (2007). Un siglo de crecimiento económico. En J. Robinson y M. Urrutia (eds.). Economía colombiana del siglo $X X$. Bogotá: Banco de la República-Fondo de Cultura Económica.

Vargas, L., Sosa, S. et al. (2012). E1 comercio como plataforma de la política exterior colombiana en la administración de Juan Manuel Santos. Colombia Internacional, 76, 259-292. 
Villar, L. y Esguerra, P. (2005). E1 comercio exterior colombiano en el siglo XX. Borradores de economia, 358, 1-53.

Williamson, J. (1990). What Washington means by policy reform. En J. Williamson (ed.). Latin American adjustment: How much has happened? (pp. 421-423). Washington DC: Institute for International Economics.

World Bank (2002). Colombia-poverty report: Main report. Washington DC: World Bank, [http://documents.worldbank.org/ curated/en/532871468770949746/Main-report]. 